\title{
Implementation of a Real-Time Distributed Network Simulator with PC-cluster
}

\author{
José R. Martí \\ Jorge A. Hollman \\ Jesús Calviño-Fraga \\ University of British Columbia \\ Electrical and Computer Engineering \\ 2356 Main Mall,Vancouver, B.C., V6T 1Z4, Canada \\ jhollman@ieee.org
}

\begin{abstract}
This paper describes the implementation of a real-time power system simulator based on a distributed cluster of PC desktop computers. A Real-time power system simulator based on a PC-cluster can successfully cope with the size requirements of growing power systems and the computational demands of fast transient studies. The distributed version of OVNI is used as core solver in addition to a developed I/O interface card. Models based on the standard tool for power systems transients simulations, the EMTP program, optimized for real time performance, assure accurate simulation results.
\end{abstract}

\section{Introduction}

The present work presents the development of a PC cluster for a real-time power system simulator. Simulations performed in a single computer for a given time step size can deal only with a maximum number of power system nodes. Unless more sophisticated solver algorithms or faster hardware are developed, this size limit restricts the areas of applicability of the single-PC simulator [8]. The PC-cluster architecture presented here overcomes this problem by allowing an indefinite number of PCs to be added to the simulator, as required, to match any power system size. Historically, an expandable PC-cluster simulator was presented in [3]. This solution was based on a general-purpose Myrinet network architecture which is relatively expensive and complex, particularly as the number of PCs in the cluster is increased. The PC-cluster solution (see Fig. 1) developed in our work takes advantage of the relatively small amount of data that needs to be transmitted between power system substation nodes connected by transmission lines. The proposed solution achieves high performance and linear scalability using an inexpensive interface card developed to fit our particular simulation strategy. This strategy is based on the concept of mapping a network of PCs to the character- istics of the power system solution network. As explained in this paper, the proposed PC-cluster simulator proves to be a solution to this limitation. In today's real-time power system operation environments, capability to simulate large systems has become the ultimate goal. The UBC OVNI ${ }^{1}$ simulator [9] can operate in real-time using a single-PC with a time step of $162.0 \mu s$ for 234 nodes/349 Branches using a Pentium II $400 \mathrm{MHz}$. With the proposed PC-cluster architecture and using the distributed version of OVNI, the same system can be simulated in real-time with a PC-cluster of five PCs Pentium II $400 \mathrm{MHz}$ with a time step of $45 \mu \mathrm{s}$. The main reason for OVNI's computational efficiency is that its solution algorithm is structured the same way as the system it is modelling. In the PC cluster layout, dense computational nodes represent the power system substations, while transmission lines connecting substations are represented by simple links to the other dense nodes. As shown in this paper, the PC-cluster concept perfectly matches the computational paradigm of OVNI and allows for easy scalability of the computational resources to match the size of the problem.

\section{Real-time Distributed Architecture}

\subsection{Analog Outputs}

In order to test external equipment such as protective relays or controllers, the voltages and currents resulting form the simulation of a given case have to be outputted as ana$\log$ signals. This task is achieved by the addition of a suitable digital to analog converter board. For this implementation of the simulator, 12-bit resolution converters were used. Since the output range of the D/A is 20 volts peak to peak, the desired outputs are scaled before they are converted. Each output is generated at every simulation time step.

Any computer in the PC-cluster may have its own D/A con-

\footnotetext{
${ }^{1}$ Object Virtual Network Integrator
} 


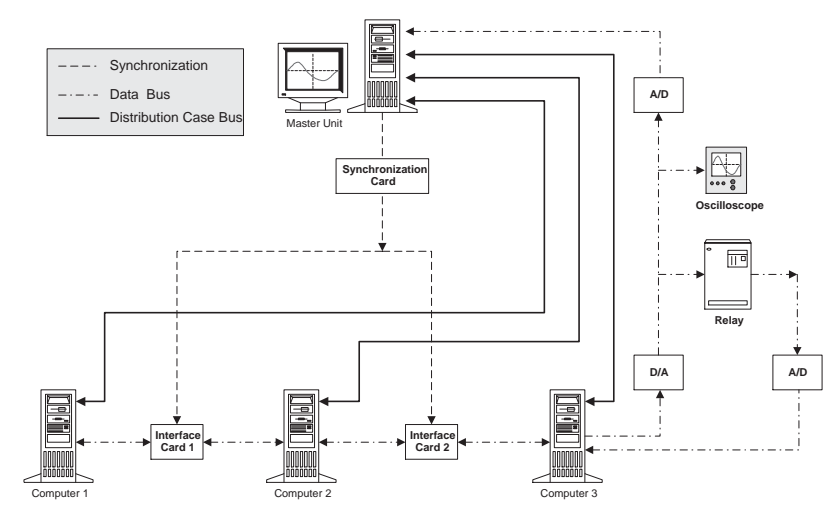

Figure 1. Proposed Architecture

verter board. As specified in [11], all outputs have to be updated at the same time. Instead of using an additional external signal to perform this synchronous update, the update of analog outputs is the first task performed at the beginning of each time step computation. Since all the solver nodes in the PC-cluster are sharing the same synchronization signal the simultaneous outputs of the desired analog signals is assured.

\subsection{Synchronization Block}

In real-time simulation all the necessary operations must be completed within the adopted time step. Since both the size of the computational load assigned to each solver node as well as the capability of each solver node to perform the subnetwork calculation can be different, it is desirable to count on an independent synchronization source. When the subsystems calculations are finished, this source is in charge of triggering a signal to all the cluster computers so that they start each individual subsystem calculation for the upcoming time step synchronically with the real time clock. The simulation program must only verify that the slowest computer in the cluster is capable of solving the system and sending the data within the time step. Then all the other computers will follow the slowest one or the one with more calculation load. Another function incorporated into the synchronization block is the start/stop signal. This signal allows the user to start and interrupt the simulation in all the machines simultaneously. The main advantage of using an external source of time is that it can be selected with the appropriate accuracy and it can be easily shared by all the computers in the parallel array. In the present implementation, a Programmable Internal Interval Timer, the Intel $82 \mathrm{C} 54$, was selected to provide the external real-time clock. The selected working mode for the I/O card design developed in this work is mode 3 , a square wave generator. The real-time source is configured through one of the parallel ports available in the master unit. An associated error will be present in the $\Delta t$, since the hardware time step is obtained through an integer value programmed into the counter when the desired time step is not an integer multiplier of $\Delta t$. However, the error introduced is not relevant (e.g. $+/-0.0625 n s)$. Another error of around $25 \mathrm{ppm} /{ }^{\circ} \mathrm{C}$ is present in the real-time clock introduced by the crystal temperature dependence. Programming of the $82 \mathrm{C} 54$ is available to the user through the simulators GUI. The $82 \mathrm{C} 54$ can be fed with a crystal of a frequency of up to $10 \mathrm{MHz}$, achieving a maximum resolution of $100 \mathrm{~ns}$ for the external timing source.

\subsection{Input/Output Interface}

To achieve real-time performance with the proposed PCcluster scheme, for a simulation time step of $50 \mu \mathrm{s}$, with 3 outputs per subsystem node, and solving the power system with the OVNI simulator, a maximum communication time of $0.45 \mu \mathrm{s} /$ byte-transferred is imposed. This setup permits the maximum distortion error in the simulation due to the Trapezoidal integration rule to be kept under $3 \%$ [4], as well as allowing the modelling of three-phase single- and double-circuit line links between subsystem nodes. The communication bandwidth is determined by the amount of data to be transferred between solver nodes. Standard input/output interfaces present in today's PCs, such as a parallel port in its configuration EPP and ECP (including the outstanding PCI-EPP/ECP interface technology) are not fast enough for the requirements of OVNI with a multi machine scheme. Even though some input/output interfaces offer higher transfer rates, these rates are valid only for very large memory blocks. This stems from the fact that these interfaces were developed mainly for video processing, massive storage devices, or video-conferencing applications in which great volumes of data need to be exchanged. In applications like OVNI, the needs are different since instead of transferring large amounts of data over a moderate time frame the objective is to transfer small memory blocks at very high speed. Because of their inherent round trip latency, in order to complete a transfer cycle, even in the case of small-sized blocks, these interfaces consume more time than the allowed step size for most of the power simulation cases.

The IDE port was chosen for several reasons: to achieve portability, to ensure the necessary transfer rate, and to follow the philosophy of using only off-the-shell computer components. Since the IDE port is available in almost all motherboards at the present time, it makes a perfect choice from the portability point of view, also allowing us to maintain a low design cost. Obtained communication rates depend on the selected operating mode and the system bus architecture. 


\subsection{Double Port Memory}

The DPM ${ }^{2}$ block is the central element in the developed I/O interface card. This block is in charge of storing and transferring data between the cluster subsystems. Each subsystem writes and reads data from different memory pages. The available memory resource for each subsystem node, using the chosen IDT7132 [5], is $16 \mathrm{~K}(2 \mathrm{~K} \times 16 \mathrm{Bit})$. The IDT7132/7142 (master and slave DPM) provides two ports with separate controls, addresses, and I/O pins that permit independent access to read or write to any location in the DPM. The implemented software for both subsystem nodes writes and reads to/from the DPM in different memory pages, and since both simulations are perfectly synchronized through the external synchronization block, there is no possibility for simultaneous access conflicts in the DPM. The DPM is available to each subsystem node at any moment, and it can be accessed simultaneously (see Fig. 2). In this implementation the simultaneous access condition is limited to different pages and access is not allowed to an individual memory cell. DPMs can be combined to form large dual port memories using master and slave memory components. In the design described here, a set of two DPMs is used in each I/O Interface card in order to obtain an array of $2 \mathrm{~K} \mathrm{x} 16$ bits memory blocks. This situation is strikingly useful due to the fact that since the $\operatorname{IDE}^{3}$ port is used, 16 bits can be written or read at any handshake without any further delay. Even though a depth expansion with this type of devices is feasible, this is not necessary for the present application.

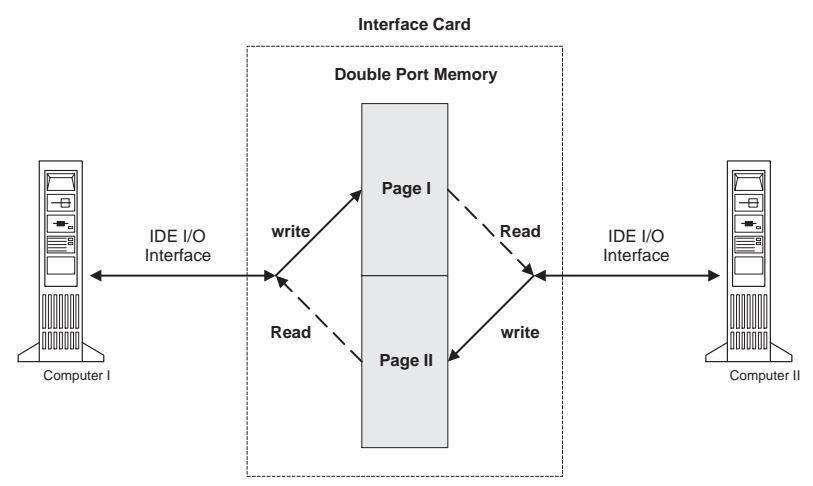

Figure 2. OVNI TCP/IP Controller

\subsection{Case Distribution}

Besides the high speed communication interface required during the real time simulation, an Ethernet network

\footnotetext{
${ }^{2}$ Double Port Memory

${ }^{3}$ Integrated Device Electronics
}

is used for remote booting of each of the solver nodes in the cluster as well as the distribution of the power system subblocks among them. The communication protocol for this function is implemented as master-slave architecture under a TCP/IP client-server scheme. Under this approach, the

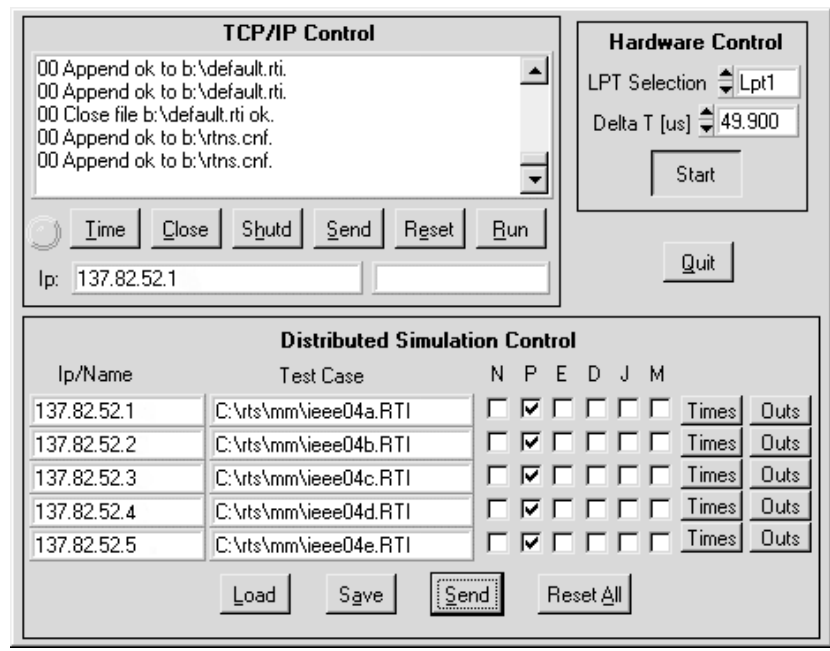

Figure 3. OVNI TCP/IP Controller

master computer, running for example Windows 95/98/NT with an appropriate user interface, sends commands to the slave solver nodes of the cluster. The data transfer protocol has been implemented in such a way that the solver nodes act as TCP/IP servers, while the master computer acts as a TCP/IP client. The master computer requests connections to each individual computer of the PC-cluster and executes any of the following commands:

- Distribute the computational load among the cluster

- Start/Stop the simulation

- Retrieve requested simulation data for plotting

The master computer also controls through the synchronization block (see Fig. 3) the simulator's time step setup, and the start/stop signal.

\section{Sample Cases: Single PC vs. PC-cluster, Real-time Performance}

A system involving a cluster of three PCs, Case I, was tested and the results are described in this section (see Fig. 4). The simulation was run first on a single PC using the OVNI solver, with the minimum possible time step needed to achieve real-time performance. After this, the same system was preprocessed to run with a PC-cluster running the OVNI distributed solver version. Two sample cases are pre- 


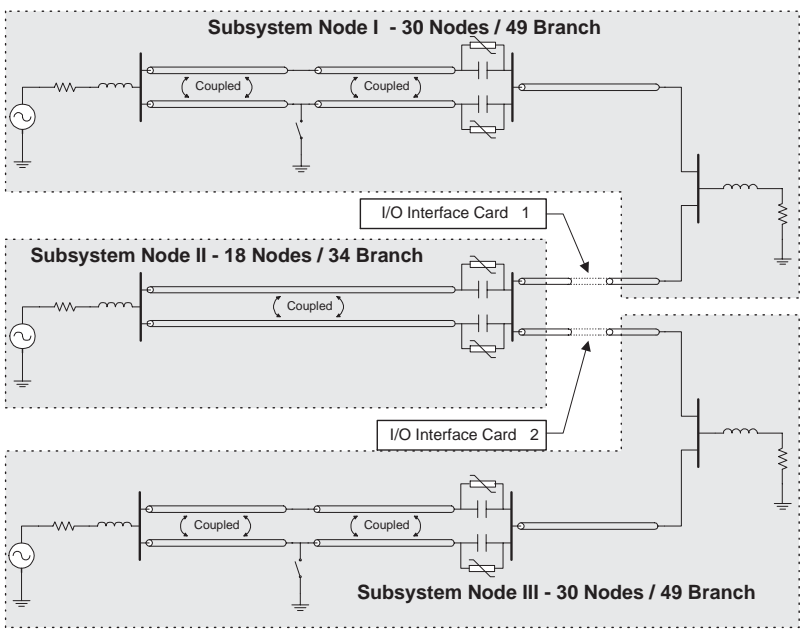

Figure 4. Sample Case I, running a PC-cluster of three PCs

sented. Both, Test Case I and Test Case II were simulated with the distributed version of OVNI solver running on Pentiums II $400 \mathrm{MHz}, 64 \mathrm{MB}$ RAM under the Phar Lap TNT ETS 9.1 real-time operating system.

Test Case I is a 78 node/132 Branch system which includes sources, three phase transmission lines featured in single and coupled double circuits, series capacitors with MOVs, and Thevenin equivalent circuits for the external parts of the network. The obtained timings for Test Case I are presented in Table 1. Test Case II consists of a 234 node/349 Branch system which includes sources, three phase transmission lines featured in single and double circuits, series capacitors with MOVs, and Thevenin equivalent circuits for the external parts of the network. The obtained timings for this case are presented in Table 2. The total solution time includes network solution plus communication times per simulation step.

When a PC-cluster solution is implemented, one of the three-phase transmission lines is chosen as the Link Line between subsystem calculation nodes (see Fig. 4). Under these circumstances, the load of the subsystem is not necessarily perfectly symmetric and does not correspond to the most effective distribution.

\section{Conclusions}

The presented work introduces an efficient and accurate PC-cluster solution for real-time network simulations using off-the-shelf equipment plus a custom-built I/O interface card for efficient communication between the nodes of the array. To this point, a PC-cluster of up to five PCs has been tested (see Fig. 5). The present OVNI software allows
Table 1. Test Case I, timing results

\begin{tabular}{|l|l|l|l|c|}
\hline Architecture & $\begin{array}{l}\text { Nodes } \\
\text { per } \\
\text { machine }\end{array}$ & $\begin{array}{l}\text { Total } \\
\text { Solution } \\
\text { Time }\end{array}$ & $\begin{array}{l}\text { Analog } \\
\text { Outputs }\end{array}$ \\
\hline \hline Single PC & 78 & $60.0 \mu \mathrm{s}$ & 6 \\
\hline \hline \multirow{3}{*}{ PC-cluster } & Node I & 30 & $33.42 \mu \mathrm{s}$ & 3 \\
\cline { 2 - 5 } & Node II & 18 & $37.77 \mu \mathrm{s}$ & 0 \\
\cline { 2 - 5 } & Node III & 30 & $33.42 \mu \mathrm{s}$ & 3 \\
\hline
\end{tabular}

Table 2. Test Case II, timing results

\begin{tabular}{|l|l|c|l|c|}
\hline \multicolumn{2}{|l|}{ Architecture } & $\begin{array}{l}\text { Nodes } \\
\text { per } \\
\text { machine }\end{array}$ & $\begin{array}{l}\text { Total } \\
\text { Solution } \\
\text { Time }\end{array}$ & $\begin{array}{l}\text { Analog } \\
\text { Outputs }\end{array}$ \\
\hline \hline Single PC & 234 & $162.0 \mu s$ & 6 \\
\hline \hline \multirow{4}{*}{ PC-cluster } & Node I & 54 & $45.34 \mu s$ & 3 \\
\cline { 2 - 5 } & Node II & 42 & $45.90 \mu s$ & 0 \\
\cline { 2 - 5 } & Node III & 42 & $45.81 \mu s$ & 0 \\
\cline { 2 - 5 } & Node IV & 42 & $45.85 \mu s$ & 0 \\
\cline { 2 - 5 } & Node V & 54 & $45.34 \mu s$ & 3 \\
\hline
\end{tabular}

communication between nodes of the PC-cluster with up to a maximum of four computers for each subsystem node, achieving real-time performance for three-phase single and double circuit links, and a basic computational load of 54 nodes per subsystem solver, within $100 \mu s$ using Pentiums II $400 \mathrm{MHz}$. The flexibility of the solution is highlighted by the fact that better performances can be achieved by just a simple CPU upgrade without performing any extra alterations to the rest of the architecture. The concept can also be perfectly applied to future faster motherboard busses with minor or no modifications.
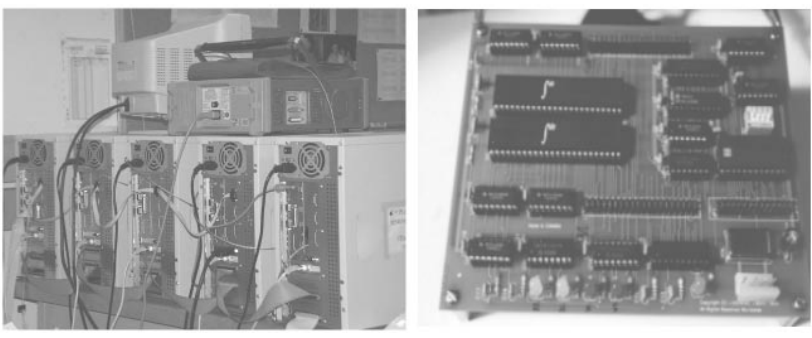

Figure 5. PC-cluster of five solver nodes \& $\mathrm{I} / \mathrm{O}$ Interface Card

\section{Acknowledgments}

This work is part of the OVNI project and is the result of the combined and previous efforts of its members. We would specially like to stress the unconditional support re- 
ceived during the completion of the presented work from Mr. L.Linares, main architect of the OVNI software.

Funding for this project proceeds from a number of sources, but particular recognition should be given to the Natural Sciences and Engineering Research Council of Canada (NSERC) program for their continued support of the OVNI research work, and to the Fundación Y.P.F. for supporting J.A.Hollman's M.A.Sc. program.

\section{References}

[1] H. Dommel. Digital computer solution of electromagnetic transient in single and multiphase networks. IEEE Transactions on Power Apparatus and Systems, PAS-88(4):388399, April 1969.

[2] H. Dommel. Introduction to the use of microtran and other emtp versions. Course Notes (ELEC 553), Dept. of Electrical \& Computer Engineering, UBC, Vancouver, B.C., Canada, 1998. available: http://www.ece.ubc.ca/power/e553.pdf.

[3] Y. Fujimoto, Y. Bin, H. Taoka, H. Tezuka, S. Sumimoto, and Y. Ishikawa. Real-time power system simulator on a pc cluster. In Proceedings of the 1998 International Conference on Power System Technology, Budapest, 1999. IPST'99.

[4] H.W.Dommel. EMTP Theory Book. Microtran Power System Analysis Corporation, 4689 W. 12th Avenue, Vancouver, B.C. V6R 2R7, Canada, 2nd edition, May 1992.

[5] IDT. Idt $7132 \&$ idt 7142 data sheet. IDT, 1999. available: http://www.idt.com/products/pages/Multi-Port-7132.html.

[6] J.A.Hollman. Real time distributed network simulation with pc clusters. Master's thesis, University of British Columbia at Electrical and Computer Engineering, Vancouver, Canada, December 1999.

[7] J.Calviño-Fraga. Implementation of a real time simulator for protective relay testing. Master's thesis, University of British Columbia at Electrical and Computer Engineering, Vancouver, Canada, June 1999.

[8] J.R.Martí and L.R.Linares. Real-time emtp-based transients simulation. IEEE Transactions on Power Apparatus and Systems, 9(3):1309-1317, August 1994.

[9] J.R.Martí, L.R.Linares, J.Calviño, J.Lin, and H.W.Dommel. Ovni: An object approach to real-time power system simulators. In Proceedings of the 1998 International Conference on Power System Technology, pages 977-981, Beijing, China, April 1998. Powercom‘98.

[10] J.R.Martí, L.R.Linares, R.Rosales, and H. Dommel. Ovni: a full-size real-time power system simulator. In International Conference on Digital Power System Simulators, pages 1309-1317, Montreal, Quebec, Canada, May 1997. ICDS'97.

[11] I. E. E. E. working group F-8 of relay input sources. Digital simulator performance requirements for relay testing. IEEE Transactions on Power Delivery, 13(1):78-84, January 1998. 